\title{
The Influence of Alkanethiols on the Production of Hydrophobic Gold Nanoparticles via Pulsed Laser Ablation in Liquids
}

\author{
Cory J. Trout ${ }^{1,2}$, Paul Kumpf ${ }^{1}$, Karli Sipps ${ }^{3}$, Julianne C. Griepenburg ${ }^{1,4}$ and Sean M. O'Malley ${ }^{1,4, *}$ \\ 1 Department of Physics, Rutgers University, Camden, NJ 08102, USA; cjt122@scarletmail.rutgers.edu (C.J.T.); \\ pck49@scarletmail.rutgers.edu (P.K.); jg1151@scarletmail.rutgers.edu (J.C.G.) \\ 2 Department of Applied Physics, Rutgers University, Newark, NJ 07102, USA \\ 3 Department of Chemistry, Rutgers University, Camden, NJ 08102, USA; ks1081@scarletmail.rutgers.edu \\ 4 Center for Computational and Integrative Biology, Rutgers University, Camden, NJ 08103, USA \\ * Correspondence: omallese@camden.rutgers.edu
}

\section{check for}

updates

Citation: Trout, C.J.; Kumpf, P.; Sipps, K.; Griepenburg, J.C.; O'Malley, S.M. The Influence of Alkanethiols on the Production of Hydrophobic Gold Nanoparticles via Pulsed Laser Ablation in Liquids. Nanomanufacturing 2021, 1, 98-108. https://doi.org/10.3390/ nanomanufacturing1030009

Academic Editors: Alina A. Manshina and Ilya Tumkin

Received: 23 July 2021

Accepted: 15 September 2021

Published: 22 September 2021

Publisher's Note: MDPI stays neutral with regard to jurisdictional claims in published maps and institutional affiliations.

Copyright: (c) 2021 by the authors. Licensee MDPI, Basel, Switzerland. This article is an open access article distributed under the terms and conditions of the Creative Commons Attribution (CC BY) license (https:// creativecommons.org/licenses/by/ $4.0 /)$.

\begin{abstract}
The ability to suspend plasmonic metal nanoparticles in apolar environments is an important feat towards harnessing their optical properties for use in amphiphilic biological environments. Pulsed laser Ablation in Liquids (PLAL) is a well-established method for the production of gold nanoparticles (AuNPs) in aqueous environments; however, ablation in organic liquids for the synthesis of hydrophobic AuNPs still has many unknowns, such as the relationship between colloidal stability and the ligand shell. In this study, hydrophobic AuNPs were produced by PLAL of gold in a 1-alkanethiol/n-decane solution and treated with laser fragmentation. Results demonstrate that longer chain length ATs produced particles with a smaller average size; however, there was no strong correlation between alkanethiol (AT) concentration and particle size. Stability was investigated by monitoring the temporal evolution of the extinction spectra which revealed that lower concentrations of AT stabilize the colloids while higher concentrations tend to result in quicker particle aggregation. Furthermore, longer chain length ATs demonstrated improved stability. Additionally, vibrational spectroscopy was employed to examine the AuNP surface chemistry, which pointed to the presence of oxidized carbon species and graphitic carbon.
\end{abstract}

Keywords: hydrophobic; gold nanoparticles; alkanethiol shell; pulsed laser ablation in liquids; photodegradation; colloidal stability

\section{Introduction}

The application of organically functionalized gold nanoparticles (AuNPs) is quite prevalent in the literature, having been found to be advantageous in the areas of biochemical sensors [1-3], drug delivery systems [4,5], and cancer theranostics [6]. Of particular interest are AuNPs functionalized with alkanethiols (AT), where the thiol covalently bonds to the gold and tethers the alkane to the AuNPs, thereby imparting a hydrophobic ligand shell. This system of alkanethiol functionalized gold has garnered particular interest due to the formation of self-assembled monolayers (SAM), where the collective van der Waals interactions between alkane chains drives their assembly into a highly ordered phase with an upright orientation [7]. In the case of AT-AuNPs in liquids, thickness and conformation of the monolayer has been shown to affect the stability and aggregation of the colloid [8,9]. The interactions that result in SAM formation can also give rise to the formation of a AuNP superlattice structure, particularly at a liquid interface [10] or on a substrate [11,12]. The proximity of the AuNPs in the lattice can lead to coupling of the localized surface plasmon resonance (LSPR), thereby altering the optical properties from that of isolated particles [13]. Additionally, as shown by Dong et al., AT-capped AuNPs can result in unexpected effects such as enhanced magnetization, which scales with chain length [14]. A 
leading benefit of AT functionality, however, still resides with the hydrophobicity it imparts to the AuNPs facilitating their suspension in apolar environments; for example, they can serve as photosensitizers in lipid and polymeric vesicles [15-17].

Bottom-up chemical methods based on the reduction of chloroauric acid are the most widely utilized approaches for gold nanoparticle synthesis [18]. These approaches include the Turkevich process-based methods for citrate capped AuNPs in water $[19,20]$ and BrustSchiffrin synthesis methods for alkanethiol-capped AuNPs in apolar solvents [21]. While such methods have been tremendously successful, the processes can be problematic as they require multiple steps and the removal of excess reactants, surfactants, and precursors.

An alternative approach for nanoparticle production that has matured rapidly in recent years is the pulsed laser ablation in liquids (PLAL) method. This process is a quasitop-down approach where a bulk metal target is submerged in liquid and irradiated by a focused laser beam, resulting in target ablation and the release of a plume containing atomic and/or clustered species that ultimately condense and grow nanoparticles [22,23]. While the processes involved are complex, the method itself is a simpler and "greener" approach as compared to chemical methods [24,25]. An additional advantage of PLAL is the ability to functionalize the NPs upon formation by simple addition of the ligand to the liquid environment [26-30]. Its common practice to follow-up the as-produced PLAL colloid with a secondary irradiation step to further reduce the size and polydispersity of the particles in a process commonly referred to as laser fragmentation [31-33].

The vast majority of PLAL-related literature has focused on laser ablation in aqueous environments, with only a few examples examining extension of the method in organic solvents such as alkanes, ethanol, acetonitrile, tetrahydrofuran, toluene, and acetone [23,34-37]. Ablation in these organic environments can produce some interesting solvent specific effects; for instance, Amendola et al. showed that graphite shells can form around AuNP cores when gold is ablated in toluene [35]. Similarly, Giorgetti et al. demonstrated enhanced stability of AuNPs when ablated in acetone due to the presence of disordered carbon forming on the particles' surface [37]. Compagnini et al. have shown that it is possible to functionalize the surface with alkanethiols while ablating in alkane/alkane-thiol solutions [29].

In this work, we build upon the understanding of nanoparticle synthesis via PLAL in organic environments by examining the formation of AuNPs in decane with the addition of different chain length alkanethiols. We study the effect of alkanethiol concentration and chain length on particle size distribution, stability, and hydrophobicity. Additionally, the occurrence of photothermal chemistry is probed along with AuNP surface modification. Colloids in the study were also subjected to laser fragmentation and data are presented prior to and following treatment.

\section{Materials and Methods}

Gold nanoparticles were synthesized by PLAL in which a (>99.9\%) pure gold plate (target) was placed in a closed stainless-steel chamber with side windows and a top optical port for the laser input. The target rested on a stainless-steel platform located within the chamber with a magnetic stirrer placed below the platform. The liquid environment was ndecane $\left(\mathrm{CH}_{3}\left(\mathrm{CH}_{2}\right)_{8} \mathrm{CH}_{3}\right)$ to which either various concentrations and / or chain lengths of nalkanethiols were added. The alkanethiols $\left(\mathrm{CH}_{3}\left(\mathrm{CH}_{2}\right)_{x} \mathrm{CH}_{2} \mathrm{SH}\right)$ studied differed only in the number of methylene groups (x) and consisted of the following: 1-octanethiol (OT, $x=8), 1$ dodecanethiol (DDT, $x=10$ ), 1-hexadecanethiol (HDT, $x=14$ ), and 1-octadecanethiol (ODT, $x=16)$. The $\mathrm{n}$-decane ( $>99 \%$ pure) was purchased from Alpha Aesar (Haverhill, MA, USA), and the OT ( $>98.5 \%$ pure), DDT ( $>98 \%$ pure), HDT ( $>95 \%$ pure), and ODT ( $>98 \%$ pure) were purchased from Millipore Sigma (Burlington, MA, USA). The decane/alkane-thiol solution $(15 \mathrm{~mL})$ was added to the chamber and the chamber was degassed for a period of $20 \mathrm{~min}$ by way of pulling vacuum on the head space above the liquid using a small diagram pump. The liquid height above the target was $13.5 \mathrm{~mm}$. This height represents a compromise between a low liquid height that reduces the path length of the focused beam 
through the colloid and a tall liquid column that lowers the laser fluence at the liquid/air interface thereby circumventing splatter on the optical port. The gold plate was irradiated using an Ekspla (Vilnius, Lithuania) model NL-303 Nd-YAG laser (5 W max. @ 1064 nm) operating at its fundamental wavelength of $1064 \mathrm{~nm}$ with a repetition rate of $10 \mathrm{~Hz}$, and a pulse duration of $4 \mathrm{~ns}$. The incident beam was focused by a $75 \mathrm{~mm}$ plano-convex lens before entering the chamber vertically through the top optical port. The pulse energy was set to $65 \mathrm{~mJ}$ and the spot size was kept at $800 \mu \mathrm{m}$ in diameter, resulting in a fluence of $13 \mathrm{~J} / \mathrm{cm}^{2}$. The spot size was determined by placing a small piece of photographic paper at the location of the target within the chamber and filling the chamber with decane to a liquid height of $13.5 \mathrm{~mm}$. The paper was then irradiated using a single pulse at five separate locations. The mean diameter of the burn spots was measured using a conventional upright reflective microscope. Irradiation occurred for a period of $4 \mathrm{~min}$ before being terminated by manual closure of the laser shutter. During this period, the beam was continuously rastered over the target surface via an xy-motorized stage traveling at $30 \mathrm{~mm} / \mathrm{min}$.

Laser fragmentation of the as-formed PLAL solution took place through the side windows of the chamber (32 $\mathrm{mm}$ path distance) and was carried out utilizing an Ekspla (Vilnius, Lithuania) 25 ps Nd-YAG laser (model PL2241,1.25 W max. @ 1064 nm) outputting at $532 \mathrm{~nm}$ with a repetition rate of $250 \mathrm{~Hz}$. The incident beam was focused via a $35 \mathrm{~mm}$ plano-convex lens with the beam entering horizontally into the chamber and the focus positioned between the two opposing side windows. The beam waist at the focus was measured in situ using the knife-edge method and found to be $1.0 \times 10^{2} \mu \mathrm{m}$. The pulse energy was set to $1.6 \mathrm{~mJ}$, and the solution was irradiated for $20 \mathrm{~min}$ while being stirred with a magnetic bar.

Extinction spectra of the colloidal solutions were collected with an Agilent Cary (Santa Clara, CA, USA) model 60 UV-Vis Spectrophotometer immediately after each process and again periodically to observe temporal evolution of the spectra. These measurements were used to gauge the stability of the colloid, where the change in absorbance at $400 \mathrm{~nm}$ was monitored over a period of $55 \mathrm{~min}$. Absorption at $400 \mathrm{~nm}$ corresponds to the interband transition in gold and therefore correlates to the amount of gold in solution [38]. The fractional change in absorbance vs. time was fit to an exponential decay and a time constant was extracted that relates to the rate of gold falling out of suspension. Attenuated total reflectance Fourier transform infrared spectroscopy (ATR-FTIR) was performed using a Bruker (Billerica, MA, USA) model ALPHA-P FT-IR spectrometer on the colloidal solutions to detect photoinduced degradation of the alkanes. The AuNP solutions were concentrated using an Eppendorf (Hamburg, Germany) Vacufuge Plus prior to dispensing the sample onto the diamond ATR crystal. Raman spectroscopy was performed using a HORIBA (Kyoto, Japan) model XplorA Plus Raman microscope with an excitation wavelength of $532 \mathrm{~nm}$. Likewise, samples were concentrated using a vacufuge prior to drop casting onto a silicon substrate; afterwards, they were placed in a vacuum chamber and allowed to dry. Transmission electron microscopy was carried out using a $200 \mathrm{keV} \mathrm{JEOL} \mathrm{(Tokyo,} \mathrm{Japan)}$ model 2010F. Samples were prepared by dropping the colloidal solution $(6 \mu \mathrm{L})$ onto Ted Pella (Redding, CA, USA) 400 mesh TEM grids containing a lacey carbon support film. The image processing program ImageJ (version 1.53c) was used to manually measure the feret diameter of the NPs. Both geometric mean and geometric standard deviation are reported given that they better describe the skewed nature of the distributions [39].

\section{Results and Discussion}

The effect of alkanethiol concentration on the size distribution and stability of AuNP colloid was investigated by a combination of UV-Vis spectroscopy and TEM. Dodecanethiol was chosen as the AT capping agent for this study given its prevalence in the literature. The concentration of DDT in the ablation liquid varied from 0 to $10 \mathrm{mM}$. Figure 1 a shows the extinction spectrum of the AuNP colloidal solutions synthesized via PLAL in a decane/DDT solution at various concentrations. The mostly monotonic absorbance increase with concentration represents a commensurate increase in the population of AuNPs within the ablation 
liquid. Note that the chamber was sonicated prior to removal of the colloid to dislodge any particles that may have physically adsorbed onto the inner surfaces of the chamber and the target. In all instances, the resonance peak is the prominent spectral feature in the UV-vis scans. As shown in Figure 1b, the LSPR position redshifts with concentration from $557 \mathrm{~nm}$ to $580 \mathrm{~nm}$, for no DDT and $10 \mathrm{mM}$, respectively. Notability, these peaks are significantly shifted to higher wavelengths and broadened compared to AuNP produced by PLAL in aqueous environments [40]. Figure $2 \mathrm{a}-\mathrm{f}$ represents the particle size distributions at each concentration. The distributions are skewed in nature which is a common feature of PLAL produced particles. A plot of the geometric mean vs. DDT concentration reveals particle sizes in the 4-6 nm range with no clear trend with concentration (see Figure $2 \mathrm{~g}$ ).
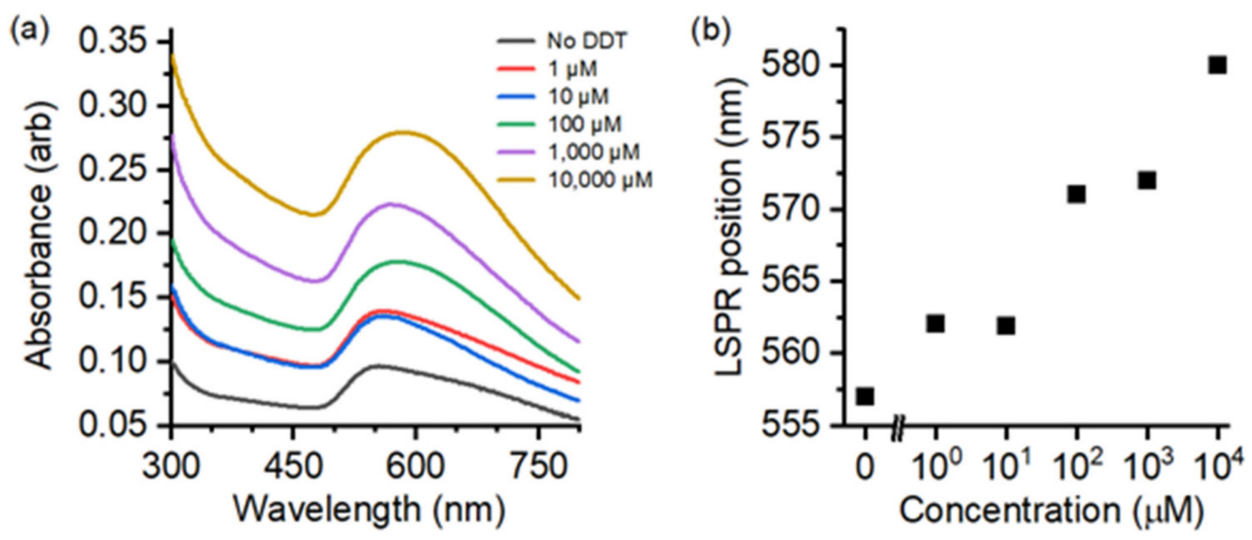

Figure 1. (a) UV/vis spectrum of AuNPs synthesized without DDT and within $1 \mu \mathrm{M}, 10 \mu \mathrm{M}, 100 \mu \mathrm{M}$, $1000 \mu \mathrm{M}$, and 10,000 $\mu \mathrm{M}$ concentrations of DDT in Decane. (b) Peak positions of the LSPR for AuNPs synthesized as a function of DDT concentrations.

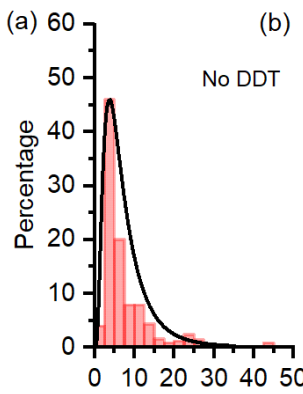

(b) 607
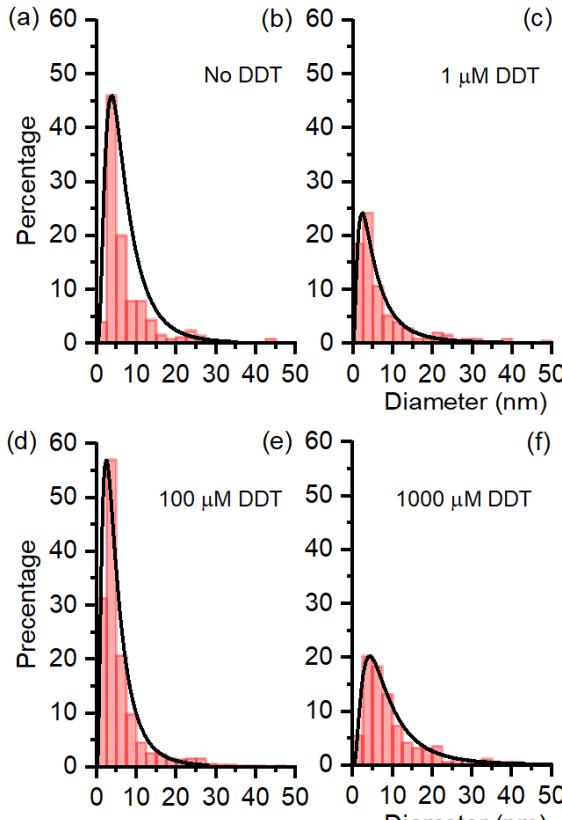

(e) 60
50
40
30
20
10
50

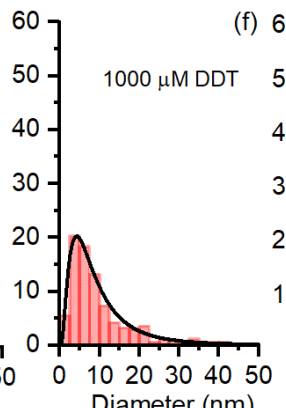

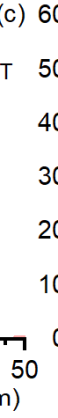

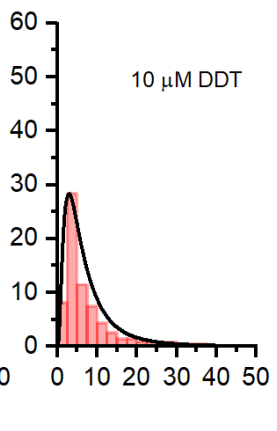

(f) 60

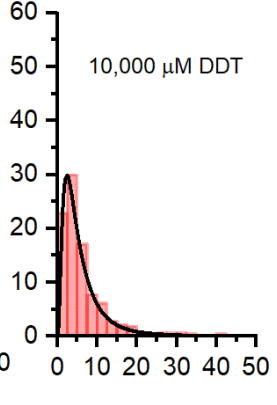

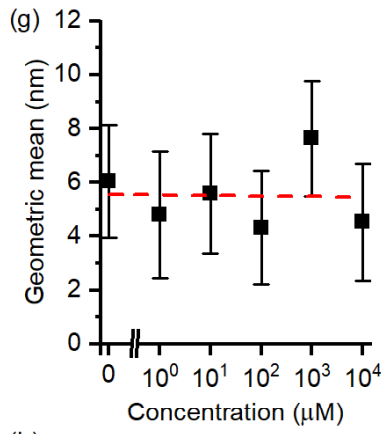

(h)

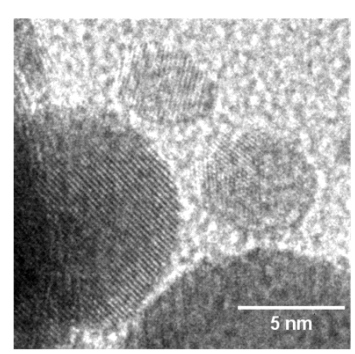

Figure 2. (a-f) Size distributions as determined via TEM for various concentrations of DDT in decane. The solid black lines represent fits to a lognormal distribution. (g) Geometric mean of the AuNPs vs. DDT concentration. (h) HRTEM image of AuNPs ablated in $10 \mathrm{mM}$ of DDT.

The lack of correlation between particle size and DDT concentration implies that the observed redshift in the plasmon resonance cannot be explained by an increase in size nor does it seem to stem from high carbon impurities within the Au lattice, as the particles display good crystallinity (see Figure $2 \mathrm{~h}$ ). It should be noted that the observed redshift is 
in contradiction to a similar study by Compagnini et al., who found a blueshift in the LSPR peak with alkanethiol concentration [29]. The discrepancy may stem from the difference in laser parameters between the studies, as slight differences in the ablation parameters can significantly affect particle characteristics [25]. Parameters for the Compagnini et al. study were $532 \mathrm{~nm}, 5 \mathrm{~ns}$, and $1 \mathrm{~J} / \mathrm{cm}^{2}$ while this work used $1064 \mathrm{~nm}, 7 \mathrm{~ns}$, and $13 \mathrm{~J} / \mathrm{cm}^{2}$. A study by Ansar et al., who chemically synthesized alkanethiol functionalized AuNPs, however, showed that the LSPR redshifts with increasing incubation time and therefore ligand coverage [41]. While an increase in surface coverage may partially explain the trend observed in Figure 1b, the primary factor contributing to the overall redshift is delocalization of the excitation brought about by aggregation, such as that seen in AuNPs destabilized by electrolytes [42].

To study the effects of chain length on the AuNP colloid, ablation took place in decane solutions containing alkanethiols that differed in the number of $\mathrm{CH}_{2}$ groups. The molar concentration was fixed at $10 \mathrm{mM}$ and all other PLAL parameters were held constant. The concentration of $10 \mathrm{mM}$ was chosen because it provides maximum ligand coverage and the greatest AuNP production rate $(0.11 \mathrm{mg} / \mathrm{min})$. In addition, the PLAL-produced colloids were subjected to laser fragmentation to reduce particle size and polydispersity. Figure 3 includes the UV/vis spectra and images for samples made with OT, DDT, HDT, and ODT both directly after ablation (Figure $3 a, c)$ and after laser fragmentation (Figure 3b,d). Figure 3a shows an increase in absorbance for the PLAL produced colloids with chain length signifying that the heightened hydrophobicity of the longer chain length ATs imparts the AuNPs with a ligand shell that enhances particle incorporation within the surrounding decane. Additionally, the increase in chain length results in a blueshift of the resonance peak from $587 \mathrm{~nm}$ for OT $(x=8)$ to $566 \mathrm{~nm}$ for ODT $(x=16)$ in a linear fashion, as shown in Figure 3e. Based on TEM, the geometric means for the PLAL colloids are $5.83 \mathrm{~nm}, 4.53 \mathrm{~nm}$, $4.63 \mathrm{~nm}$, and $3.45 \mathrm{~nm}$ for OT, DDT, HDT, and ODT, respectively, hence displaying a general decrease with increasing chain length.

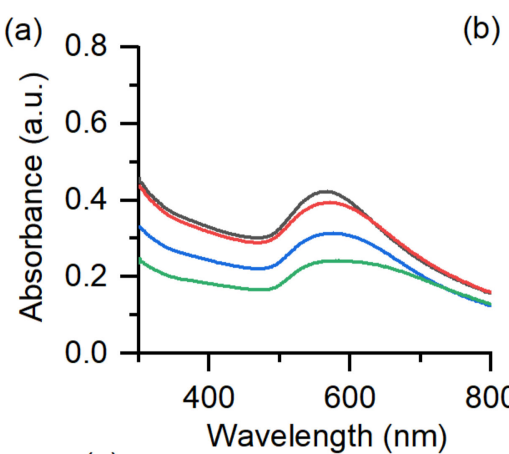

(c)

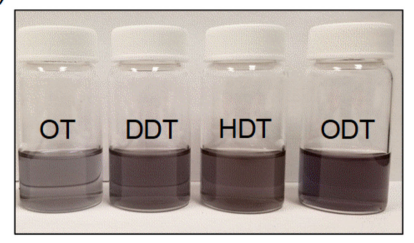

(b)

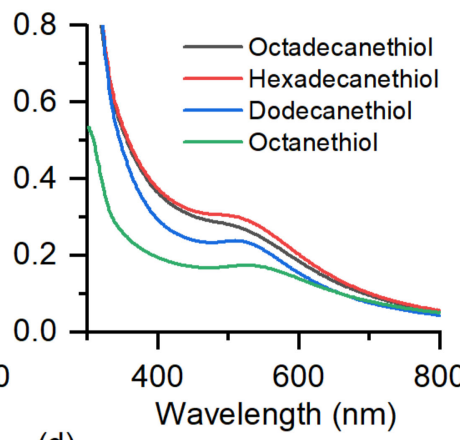

(d)

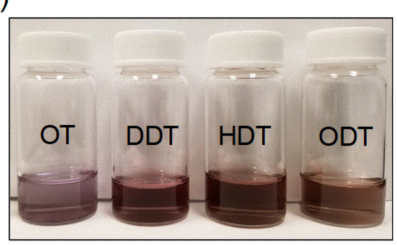

(e)

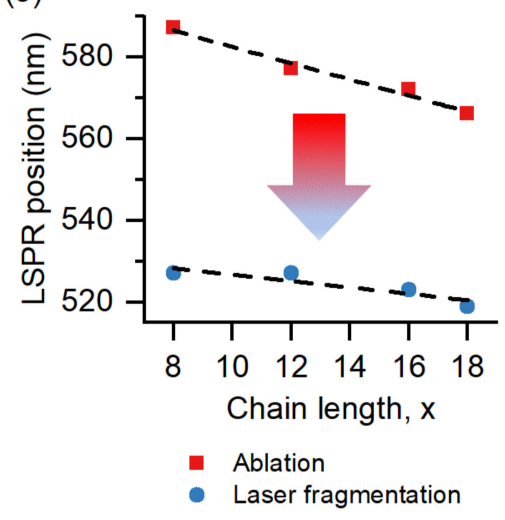

Figure 3. (a) UV-vis spectra of AuNP solutions synthesized in $10 \mathrm{mM}$ OT (green), DDT (blue), HDT (red), and ODT (black) after ablation, and (b) after laser fragmentation. Images of colloidal solutions taken after (c) ablation and (d) laser fragmentation. (e) LSRP peak position as a function of alkanethiol chain length for colloids formed after ablation (red) and laser fragmentation (blue). The $x$-axis signifies the number of $\mathrm{CH}_{2}$ units.

As shown in Figure 3b, treatment of the colloids by laser fragmentation resulted in weaker LSPR peaks and a pronounced blueshift (Figure 3e), pointing to the successful fragmentation of the particles. TEM reveals a drastic reduction in size and narrowing of the distribution, as shown in Figure 4a. These results are consistent with fragmentation 
of the AuNPs in aqueous environments [31,43]. Chain length, however, did not strongly influence the final size, where the mean particle sizes were all around $2.6 \mathrm{~nm}$ (Figure $4 \mathrm{~b}$ ).
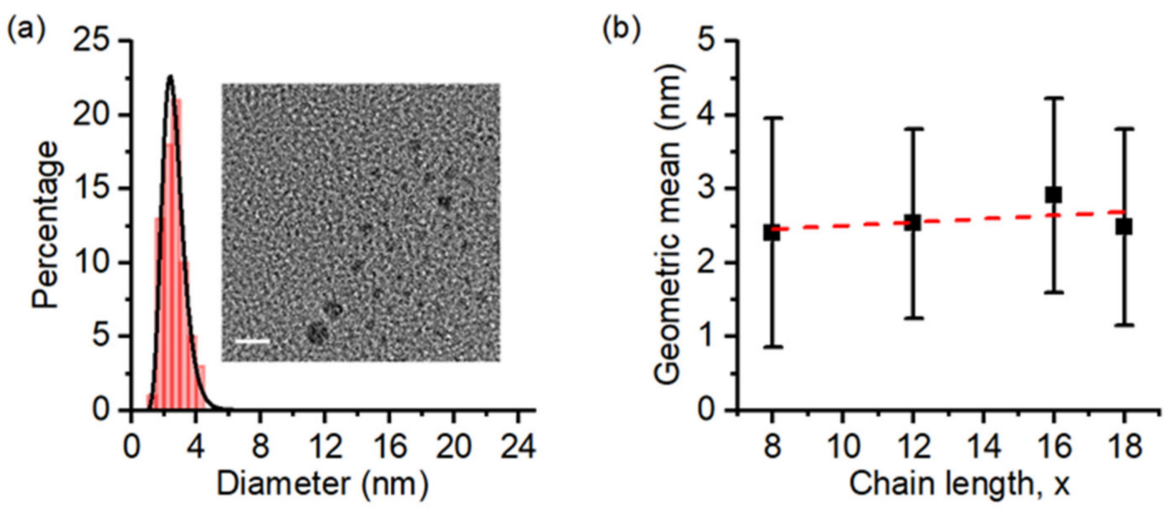

Figure 4. (a) Size distribution for AuNPs functionalized with DDT and subjected to laser fragmentation. The inset is of a representative TEM image where the scale bar represents $5 \mathrm{~nm}$. (b) Geometric mean vs. alkanethiol chain for AuNPs subjected to secondary irradiation.

To gauge the hydrophobicity of the AuNPs after laser fragmentation, the particles were resuspended in three different liquids (water, ethanol, and decane). Only those particles produced in $10 \mathrm{mM}$ DDT were chosen for the resuspension study. The colloidal samples were vacufuged until a solid pellet remained at the bottom of Eppendorf tubes, then filled with the respective liquids, and finally sonicated for $5 \mathrm{~min}$. As shown in Figure 5, particles resuspended in water and ethanol preferentially adhered to the walls of the tubes; however, NPs resuspended in decane resulted in a stable colloid. After resuspension, TEM analysis revealed no adverse effects on particle size.
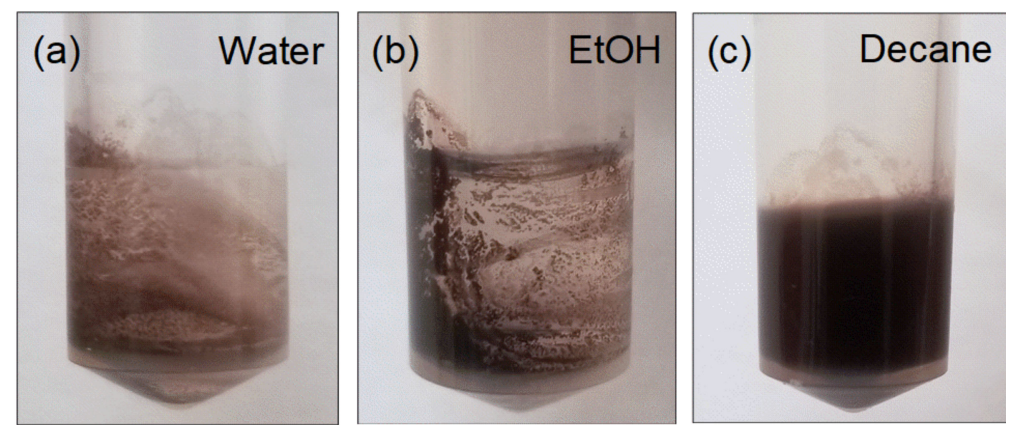

Figure 5. Images of AuNPs resuspended in (a) DDI water, (b) EtOH, and (c) decane.

Though there are several instances of carbon deposits forming on the surface of nanoparticles produced by PLAL in organic liquids [37,44-46], the occurrence of such photothermal chemistry has, for the large part, been overlooked in PLAL studies involving functionalized NP synthesis. Therefore, a combination of FTIR and Raman spectroscopy was utilized to investigate the occurrence of degradation of the ablation medium and/or the presence of deposits on the surface of the AuNPs. Figure 6a is a comparison of ATRFTIR spectra taken from a stock solution of $10 \mathrm{mM}$ DDT in decane to that of unconcentrated and concentrated colloidal solutions of AuNPs produced by PLAL and subjected to laser fragmentation. The term "unconcentrated" refers to an as-produced colloidal solution while "concentrated" refers to a sample where the concentration of AuNPs has been increased by a factor of 1000 through decane evaporation of the sample using a vacufuge. Both the spectra for the stock solution and the unconcentrated colloid show excellent overlap, indicating that the ablation liquid did not undergo significant degradation during either PLAL or laser fragmentation. The concentrated sample, however, contains two small peaks at $1645 \mathrm{~cm}^{-1}$ and $1712 \mathrm{~cm}^{-1}$. These peaks correspond to stretching modes of 
double bonded carbon found in alkenes and that of a carbonyl, respectively. The absence of these peaks from the unconcentrated AuNP solution suggests that these components are associated with compounds coating the AuNP surface, as the concentrated sample would enhance any signal originating from the particles.
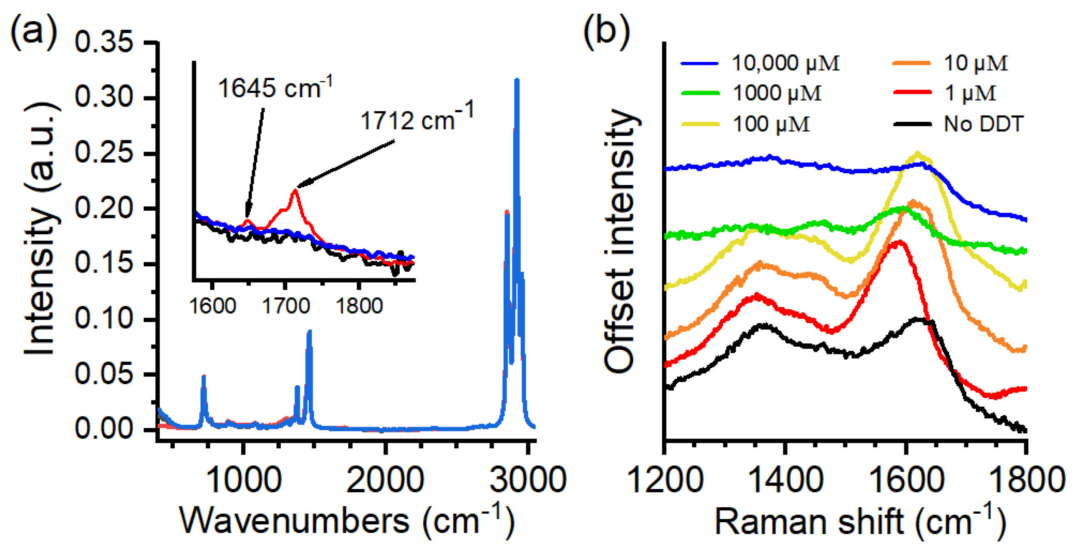

Figure 6. (a) ATR-FTIR spectrum of $10 \mathrm{mM}$ DDT in decane (black), AuNP solution synthesized with $10 \mathrm{mM}$ DDT (blue), and a concentrated solution of AuNPs synthesized in $10 \mathrm{mM}$ DDT (red). The insert zooms in on the range from 1575 to $1875 \mathrm{~cm}^{-1}$. (b) Raman spectra of AuNPs synthesized with various concentrations of DDT. Note that the spectra are vertically offset for clarity.

The transformation of widely available hydrocarbons, such as alkanes, to their oxygenated or dehydrogenated state is a well-studied area [47-49]. Plasmonic nanoparticles, such as AuNPs, have shown promise in this field where the LSPR catalyzes these reactions by enhancing photon absorption and exciton generation [50-52]. Therefore, it is reasonable to assume that laser fragmentation at $532 \mathrm{~nm}$ leads to plasmonic excitation of the colloidal AuNPs that brings about molecular modification, resulting in the presence of alkene and ketone containing compounds on the surface of the AuNPs.

To reduce the oxidation of the alkane chains, all ablations and irradiations took place in a degassed ablation liquid under continuous vacuum being pulled on the head space above the liquid. While molecular oxygen does not readily dissolve in decane, carbon dioxide does have a propensity for absorption in hydrocarbon [53,54]. To determine the effectiveness of this precaution, a comparative study was carried out between AuNPs synthesized in decane solution open to atmosphere vs. degassed solution under vacuum. Despite degassing and performing the ablation under moderate vacuum ( -26 inHg), FTIR analysis indicates only a slight $(\sim 5 \%)$ reduction in carbonyl. This qualitative estimate was realized via a ratiometric comparison of the carbonyl signal to the $\mathrm{C}-\mathrm{H}$ scissoring mode of the alkane chain at $1465 \mathrm{~cm}^{-1}$ [22].

In addition, Raman spectroscopy was performed on pelletized AuNPs samples made with DDT at various concentrations ranging from 0 to $10 \mathrm{mM}$. The spectra, shown in Figure $6 \mathrm{~b}$, focuses on the region associated with graphitic carbon, i.e., the disordered (D) and graphite $(G)$ bands $[55,56]$. Samples synthesized from 0 to $100 \mu$ M DDT contain clear D and $G$ peaks centered at $1355 \mathrm{~cm}^{-1}$ and $1592 \mathrm{~cm}^{-1}$, respectively. However, both the $1 \mathrm{mM}$ and $10 \mathrm{mM}$ samples show significantly reduced $\mathrm{G}$ and D signals. Formation of graphitic carbon takes place from the physical interaction of hydrocarbon species, e.g., decane or the alkane chains of DDT, with the hot surface of the nanoparticle following thermalization. Given this, the observed reduction in deposited carbon at the higher concentrations most likely stems from a more complete thiol ligand shell which serves to shield the alkenes from physically encountering the gold surface.

Colloidal stability is an important parameter for a wide range of applications including conductive inks $[57,58]$ and color-improving additives for LEDs $[59,60]$. Hence, the stability of the AT functionalized AuNPs colloids were investigated to gain insight into the relationship between the ablation liquid, functionalizing ligands, and the gold 
nanoparticles. Classical colloid theory assumes radially uniform core-shell structures, and as such, the interaction potentials between adjacent particles are also taken to be radially symmetric. These assumptions, however, can cause the theory to fall short in predicting the stability of some colloidal systems, such as those in this study. For example, Monego et al. used a combined experimental and molecular dynamic simulation approach to demonstrate that classical colloid theory fails to describe the stability of apolar nanoparticles in apolar solvents due to it neglecting to account for ordering of the ligand shell around the nanoparticle core [9]. In the case of nanoparticles below a threshold size, the ligands can preferentially arrange into ordered bundles forming an anisotropic shell. Smaller particles have an increased radius of curvature and therefore, the volume occupied by the ligand heads is much smaller than that of the ligand tails, which disrupts long range ordering of adjacent alkane chains. It is energetically favorable for the ordered bundles of adjacent particles to interlock, analogous to the interlocking of two gears. Hence, these anisotropic shells can produce attractive interparticle potentials that promote agglomeration in certain regimes and thereby contradict predictions made by classical colloidal theory $[8,9]$.

The short-term stability (fast sedimentation component) of the colloids was analyzed by monitoring the temporal decrease in absorbance at $400 \mathrm{~nm}$ for a 55 -minute period preceding PLAL synthesis. The fractional change in absorbance was then fit using the following expression: $f=A \exp \left(\frac{-t}{\tau}\right)+B$, where $\tau$ is the characteristic time constant for those components of the colloid that experience fast sedimentation while $B$ accounts for components with much slower sedimentation rates. The time constant was extracted for DDT concentrations ranging from $1 \mu \mathrm{M}$ to $10 \mathrm{mM}$ (see Figure 7a,b). Note that neat decane was omitted due to a tendency for the AuNPs to adhere to the walls of the cuvette.

(a)

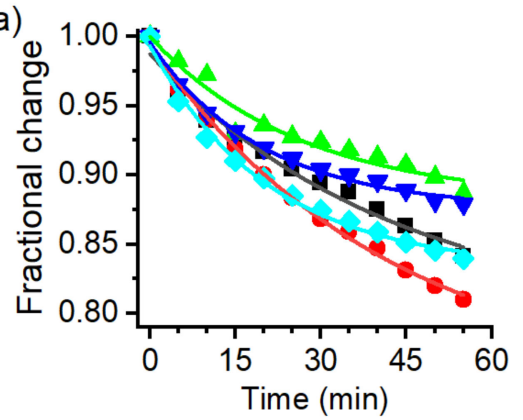

(b)

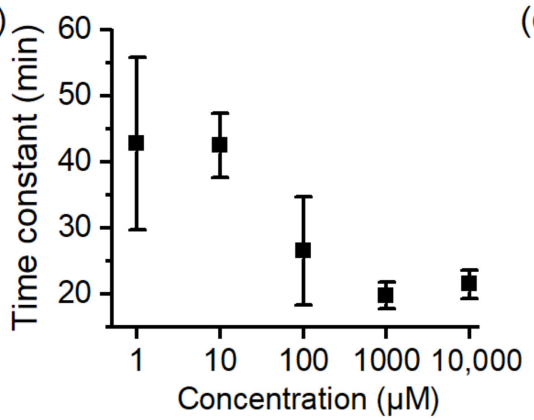

(c)

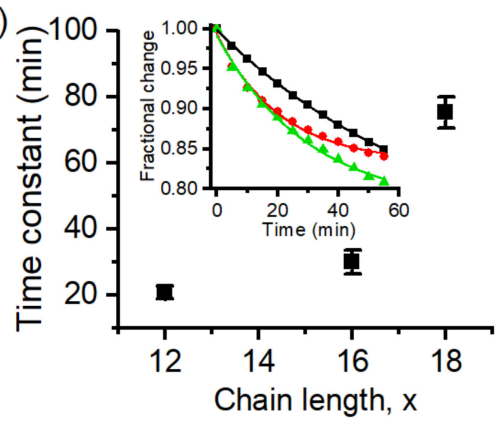

Figure 7. (a) Absorbance at $400 \mathrm{~nm}$ versus time, fitted to an exponential decay, for DDT concentrations of $1 \mu \mathrm{M}$ (black), $10 \mu \mathrm{M}$ (red), $100 \mu \mathrm{M}$ (green), $1000 \mu \mathrm{M}$ (blue), and 10,000 $\mu \mathrm{M}$ (cyan). (b) Time constant values for components experiencing fast sedimentation versus DDT concentration. (c) Time constants associated with components experiencing fast sedimentation versus alkanethiol chain length. The inset depicts the absorbance at $400 \mathrm{~nm}$ vs. time to an exponential function with DDT (red), HDT (green), and ODT (black).

As shown, particles produced with higher concentrations of DDT exhibit a shorter time constant as compared to those at lower concentrations. Such behavior could be explained as follows: as depicted in Figure $2 \mathrm{~g}$, regardless of concentration, the mean sizes of the particles are below $8 \mathrm{~nm}$ and thus are generally considered "small". These small AuNPs may experience weak Van der Waals interactions between their gold cores and as such, the tendency to aggregate is low. The adsorption of AT at low concentrations promotes stability within the hydrophobic environment of the decane; however, at higher concentrations, the AT surface coverage is sufficient, such that attractive ligand-ligand interactions between adjacent particles leads to aggregation and, ultimately, reduced stability.

Additionally, the colloidal stability of AuNPs synthesized in $10 \mathrm{mM}$ DDT, HDT, and ODT was examined by the same method. Particles produced with OT were excluded from this study due to their adhesion to the walls of the cuvette. Figure 7 reveals that as the chain length of alkanethiol increases, the stability of the colloid increases, as well. It should be noted that all three samples fall out of solution in approximately $24 \mathrm{~h}$; however, the suspension can easily be restored with agitation. After treatment with laser fragmentation, 
colloidal solutions synthesized in 10 mM DDT, HDT, and ODT remain stable indefinitely, which can be attributed to their significantly smaller sizes and reduced polydispersity. A noted exception are AuNPs functionalized with OT, as they are still unstable even after laser fragmentation and either fall out of solution or adhere to the walls of the vial, the likely factor being the low hydrophobicity imparted to the AuNPs due to the short chain length of octanethiol.

\section{Conclusions}

In conclusion, hydrophobic gold nanoparticles with a nominal size of $5.5 \mathrm{~nm}$ were successfully synthesized via the ablation of a gold target immersed in a decane/alkanethiol environment. An increase in alkanethiol concentration was shown to increase particle aggregation and reduce stability of the colloid, which is likely brought about by attractive interactions between alkane chains on the surface of neighboring particles. Overall stability was found to improve with increased chain length. Additionally, laser fragmentation of the colloidal solutions at $532 \mathrm{~nm}$ was shown to further reduce particle size and polydispersity with no photoinduced degradation of the bulk liquid nor adverse effects on the hydrophobicity of the particles. Vibrational spectroscopy on highly concentrated samples revealed that the surface of the AuNPs produced weak signals associated with oxidized carbon species and graphitic carbon. The presence of graphitic carbon was shown to be suppressed at higher alkanethiol concentrations where the robust thiol shell serves as barrier between alkane species and the excited nanoparticles.

Author Contributions: Conceptualization, C.J.T., J.C.G., and S.M.O.; methodology, C.J.T., J.C.G., and S.M.O.; formal analysis, C.J.T. and S.M.O.; investigation, C.J.T., P.K., and K.S.; resources, S.M.O. and J.C.G.; data curation, C.J.T., P.K., and S.M.O.; writing-original draft preparation, C.J.T. and S.M.O.; writing-C.J.T., J.C.G., and S.M.O.; visualization, C.J.T. and S.M.O.; supervision, C.J.T., S.M.O.; project administration, S.M.O. All authors have read and agreed to the published version of the manuscript.

Funding: This research received no external funding.

Institutional Review Board Statement: Not applicable.

Informed Consent Statement: Not applicable.

Data Availability Statement: The data presented in this study are available on request from the corresponding author.

Acknowledgments: The Authors would like to thank V.M.O. for help with the graphical abstract.

Conflicts of Interest: The authors declare no conflict of interest.

\section{References}

1. Lee, J.-H.; Cho, H.-Y.; Choi, H.K.; Lee, J.-Y.; Choi, J.-W. Application of Gold Nanoparticle to Plasmonic Biosensors. Int. J. Mol. Sci. 2018, 19, 2021. [CrossRef] [PubMed]

2. Zeng, S.; Yong, K.-T.; Roy, I.; Dinh, X.Q.; Yu, X.; Luan, F. A Review on Functionalized Gold Nanoparticles for Biosensing Applications. Plasmonics 2011, 6, 491-506. [CrossRef]

3. Li, Y.; Schluesener, H.J.; Xu, S. Gold nanoparticle-based biosensors. Gold Bull 2010, 43, 29-41. [CrossRef]

4. Jeong, E.H.; Jung, G.; Hong, C.A.; Lee, H. Gold nanoparticle (AuNP)-Based drug delivery and molecular imaging for biomedical applications. Arch. Pharm. Res. 2014, 37, 53-59. [CrossRef] [PubMed]

5. Rana, S.; Bajaj, A.; Mout, R.; Rotello, V.M. Monolayer coated gold nanoparticles for delivery applications. Adv. Drug Deliv. Rev. 2012, 64, 200-216. [CrossRef]

6. Liang, H.; Tian, H.; Deng, M.; Chen, X. Gold Nanoparticles for Cancer Theranostics. Chin. J. Chem. 2015, 33, 1001-1010. [CrossRef]

7. Ulman, A. Formation and Structure of Self-Assembled Monolayers. Chem. Rev. 1996, 96, 1533-1554. [CrossRef]

8. Kister, T.; Monego, D.; Mulvaney, P.; Widmer-Cooper, A.; Kraus, T. Colloidal Stability of Apolar Nanoparticles: The Role of Particle Size and Ligand Shell Structure. ACS Nano 2018, 12, 5969-5977. [CrossRef]

9. Monego, D.; Kister, T.; Kirkwood, N.; Mulvaney, P.; Widmer-Cooper, A.; Kraus, T. Colloidal Stability of Apolar Nanoparticles: Role of Ligand Length. Langmuir 2018, 34, 12982-12989. [CrossRef]

10. Raveendran, A.; DeWolf, C.E.; Bu, W.; McWhirter, S.; Meron, M.; Lin, B.; Meli, M.-V. Langmuir Films of n-Alkanethiol-Capped Gold Nanoparticles and n-Alkanes: Interfacial Mixing Scenarios Assessed by X-ray Reflectivity and Grazing Incidence Diffraction. J. Phys. Chem. C 2018, 122, 2975-2982. [CrossRef] 
11. Martin, J.E.; Wilcoxon, J.P.; Odinek, A.J.; Provencio, P. Control of the Interparticle Spacing in Gold Nanoparticle Superlattices. J. Phys. Chem. B 2000, 104, 9475-9486. [CrossRef]

12. Kanehara, M.; Oumi, Y.; Sano, T.; Teranishi, T. Formation of Low-Symmetric 2D Superlattices of Gold Nanoparticles through Surface Modification by Acid-Base Interaction. J. Am. Chem. Soc. 2003, 125, 8708-8709. [CrossRef]

13. Chen, C.-F.; Tzeng, S.-D.; Chen, H.-Y.; Lin, K.-J.; Gwo, S. Tunable Plasmonic Response from Alkanethiolate-Stabilized Gold Nanoparticle Superlattices: Evidence of Near-Field Coupling. J. Am. Chem. Soc. 2008, 130, 824-826. [CrossRef] [PubMed]

14. Dong, P.; Fisher, E.A.; Meli, M.-V.; Trudel, S. Tuning the magnetism of gold nanoparticles by changing the thiol coating. Nanoscale 2020, 12, 19797-19803. [CrossRef]

15. Mathiyazhakan, M.; Wiraja, C.; Xu, C. A Concise Review of Gold Nanoparticles-Based Photo-Responsive Liposomes for Controlled Drug Delivery. Nano-Micro Lett. 2018, 10, 1-10. [CrossRef]

16. DiSalvo, G.M.; Robinson, A.R.; Aly, M.S.; Hoglund, E.R.; O'Malley, S.M.; Griepenburg, J.C. Polymersome Poration and Rupture Mediated by Plasmonic Nanoparticles in Response to Single-Pulse Irradiation. Polymers 2020, 12, 2381. [CrossRef] [PubMed]

17. Trout, C.J.; Clapp, J.A.; Griepenburg, J.C. Plasmonic carriers responsive to pulsed laser irradiation: A review of mechanisms, design, and applications. New J. Chem. 2021. [CrossRef]

18. Zhou, J.; Ralston, J.; Sedev, R.; Beattie, D.A. Functionalized gold nanoparticles: Synthesis, structure and colloid stability. J. Colloid Interface Sci. 2009, 331, 251-262. [CrossRef] [PubMed]

19. Kimling, J.; Maier, M.; Okenve, B.; Kotaidis, V.; Ballot, H.; Plech, A. Turkevich Method for Gold Nanoparticle Synthesis Revisited. J. Phys. Chem. B 2006, 110, 15700-15707. [CrossRef]

20. Schulz, F.; Homolka, T.; Bastús, N.G.; Puntes, V.; Weller, H.; Vossmeyer, T. Little Adjustments Significantly Improve the Turkevich Synthesis of Gold Nanoparticles. Langmuir 2014, 30, 10779-10784. [CrossRef]

21. Perala, S.R.K.; Kumar, S. On the Mechanism of Metal Nanoparticle Synthesis in the Brust-Schiffrin Method. Langmuir 2013, 29, 9863-9873. [CrossRef]

22. Tomko, J.; O’Malley, S.M.; Trout, C.; Naddeo, J.; Jimenez, R.; Griepenburg, J.C.; Soliman, W.; Bubb, D.M. Cavitation bubble dynamics and nanoparticle size distributions in laser ablation in liquids. Colloids Surf. A Physicochem. Eng. Asp. 2017, 522, 368-372. [CrossRef]

23. Zhang, D.; Gökce, B.; Barcikowski, S. Laser Synthesis and Processing of Colloids: Fundamentals and Applications. Chem. Rev. 2017, 117, 3990-4103. [CrossRef]

24. Amendola, V.; Meneghetti, M. Laser ablation synthesis in solution and size manipulation of noble metal nanoparticles. Phys. Chem. Chem. Phys. 2009, 11, 3805-3821. [CrossRef] [PubMed]

25. Amendola, V.; Meneghetti, M. What controls the composition and the structure of nanomaterials generated by laser ablation in liquid solution? Phys. Chem. Chem. Phys. 2013, 15, 3027-3046. [CrossRef] [PubMed]

26. Petersen, S.; Jakobi, J.; Hörtinger, A.; Barcikowski, S. In situ conjugation-Tailored nanoparticle-conjugates by laser ablation in liquids. J. Laser Micro Nanoeng 2009, 4, 71-74. [CrossRef]

27. Walter, J.G.; Petersen, S.; Stahl, F.; Scheper, T.; Barcikowski, S. Laser ablation-based one-step generation and bio-functionalization of gold nanoparticles conjugated with aptamers. J. Nanobiotechnol. 2010, 8, 21. [CrossRef] [PubMed]

28. Barcikowski, S.; Jakobi, J.; Petersen, S.; Hahn, A.; Bärsch, N.; Chichkov, B. Adding functionality to metal nanoparticles during femtosecond laser ablation in liquids. ICALEO 2007, 404. [CrossRef]

29. Compagnini, G.; Scalisi, A.A.; Puglisi, O.; Spinella, C. Synthesis of gold colloids by laser ablation in thiol-alkane solutions. J. Mater. Res. 2004, 19, 2795-2798. [CrossRef]

30. Letzel, A.; Reich, S.; Rolo, T.D.S.; Kanitz, A.; Hoppius, J.; Rack, A.; Olbinado, M.P.; Ostendorf, A.; Gokce, B.; Plech, A.; et al. Time and Mechanism of Nanoparticle Functionalization by Macromolecular Ligands during Pulsed Laser Ablation in Liquids. Langmuir 2019, 35, 3038-3047. [CrossRef]

31. Kurita, H.; Takami, A.; Koda, S. Size reduction of gold particles in aqueous solution by pulsed laser irradiation. Appl. Phys. Lett. 1998, 72, 789-791. [CrossRef]

32. González-Rubio, G.; Guerrero-Martínez, A.; Liz-Marzán, L.M. Reshaping, Fragmentation, and Assembly of Gold Nanoparticles Assisted by Pulse Lasers. Acc. Chem. Res. 2016, 49, 678-686. [CrossRef]

33. Fales, A.M.; Vogt, W.C.; Pfefer, T.J.; Ilev, I.K. Quantitative Evaluation of Nanosecond Pulsed Laser-Induced Photomodification of Plasmonic Gold Nanoparticles. Sci. Rep. 1570. [CrossRef]

34. Amendola, V.; Polizzi, S.; Meneghetti, M.; Amendola, V. Laser ablation synthesis of gold nanoparticles in organic solvents. J. Phys. Chem. B 2006, 110, 7232-7237. [CrossRef]

35. Amendola, V.; Rizzi, G.A.; Polizzi, S.; Meneghetti, M. Synthesis of Gold Nanoparticles by Laser Ablation in Toluene: Quenching and Recovery of the Surface Plasmon Absorption. J. Phys. Chem. B 2005, 109, 23125-23128. [CrossRef] [PubMed]

36. Compagnini, G.; Scalisi, A.A.; Puglisi, O. Production of gold nanoparticles by laser ablation in liquid alkanes. J. Appl. Phys. 2003, 94, 7874-7877. [CrossRef]

37. Giorgetti, E.; Muniz-Miranda, M.; Marsili, P.; Scarpellini, D.; Giammanco, F. Stable gold nanoparticles obtained in pure acetone by laser ablation with different wavelengths. J. Nanopart. Res. 2012, 14, 1-13. [CrossRef]

38. Hendel, T.; Wuithschick, M.; Kettemann, F.; Birnbaum, A.; Rademann, K.; Polte, J. In Situ Determination of Colloidal Gold Concentrations with UV-Vis Spectroscopy: Limitations and Perspectives. Anal. Chem. 2014, 86, 11115-11124. [CrossRef] [PubMed] 
39. Granqvist, C.G.; Buhrman, R.A. Ultrafine metal particles. J. Appl. Phys. 1976, 47, 2200-2219. [CrossRef]

40. He, Y.Q.; Liu, S.P.; Kong, L.; Liu, Z.F. A study on the sizes and concentrations of gold nanoparticles by spectra of absorption, resonance Rayleigh scattering and resonance non-linear scattering. Spectrochim. Acta Part A Mol. Biomol. Spectrosc. 2005, 61, 2861-2866. [CrossRef]

41. Ansar, S.M.; Gadogbe, M.; Siriwardana, K.; Howe, J.Y.; Dogel, S.; Hosseinkhannazer, H.; Collier, W.E.; Rodriguez, J.M.D.; Zou, S.; Zhang, D. Dispersion Stability, Ligand Structure and Conformation, and SERS Activities of 1-Alkanethiol Functionalized Gold and Silver Nanoparticles. J. Phys. Chem. C 2014, 118, 24925-24934. [CrossRef]

42. Wang, G.; Sun, W. Optical Limiting of Gold Nanoparticle Aggregates Induced by Electrolytes. J. Phys. Chem. B 2006, 110, 20901-20905. [CrossRef]

43. Takami, A.; Kurita, H.; Koda, S. Laser-Induced Size Reduction of Noble Metal Particles. J. Phys. Chem. B 1999, 103, 1226-1232. [CrossRef]

44. Amendola, V.; Polizzi, S.; Meneghetti, M. Free Silver Nanoparticles Synthesized by Laser Ablation in Organic Solvents and Their Easy Functionalization. Langmuir 2007, 23, 6766-6770. [CrossRef] [PubMed]

45. Yu, X.; Terakawa, S.; Hayashi, S.; Asaka, T.; Itoigawa, F.; Ono, S.; Takayanagi, J. Carbonization of Silicon Nanoparticles via Ablation Induced by Femtosecond Laser Pulses in Hexane. Arab. J. Sci. Eng. 2017, 42, 4221-4226. [CrossRef]

46. Matsue, T.; Yamada, Y.; Kobayashi, Y. Iron carbide nanoparticles produced by laser ablation in organic solvent. Hyperfine Interac. 2012, 205, 31-35. [CrossRef]

47. Kumar, A.; Kumar, V.P.; Srikanth, A.; Vishwanathan, V.; Chary, K.V.R. Vapor Phase Oxidation of Benzyl Alcohol over Nano Au/SBA-15 Catalysts: Effect of Preparation Methods. Catal. Lett. 2015, 146, 35-46. [CrossRef]

48. Conte, M.; Miyamura, H.; Kobayashi, S.; Chechik, V. Spin Trapping of Au-H Intermediate in the Alcohol Oxidation by Supported and Unsupported Gold Catalysts. J. Am. Chem. Soc. 2009, 131, 7189-7196. [CrossRef]

49. Mitsudome, T.; Noujima, A.; Mizugaki, T.; Jitsukawa, K.; Kaneda, K. Efficient Aerobic Oxidation of Alcohols using a HydrotalciteSupported Gold Nanoparticle Catalyst. Adv. Synth. Catal. 2009, 351, 1890-1896. [CrossRef]

50. Zou, X.; Tao, Z.; Asefa, T. Semiconductor and Plasmonic Photocatalysis for Selective Organic Transformations. Curr. Org. Chem. 2013, 17, 1274-1287. [CrossRef]

51. Takeuchi, Y.; Fujita, T.; Takeyasu, N. Plasmon-mediated chemical transformation from alkane to alkene on a silver nanoparticle array under $532 \mathrm{~nm}$ excitation. Phys. Chem. Chem. Phys. 2019, 21, 7502-7507. [CrossRef]

52. Hallett-Tapley, G.L.; Silvero, M.J.; González-Béjar, M.; Grenier, M.; Netto-Ferreira, J.C.; Scaiano, J.C. Plasmon-Mediated Catalytic Oxidation of sec-Phenethyl and Benzyl Alcohols. J. Phys. Chem. C 2011, 115, 10784-10790. [CrossRef]

53. Battino, R. (Ed.) Oxygen and Ozone, In IUPAC Solubility Data Series; Pergamon Press: Oxford, UK, 1981 ; Volume 7.

54. Fogg, P.G.T. (Ed.) Carbon Dioxide in Non-Aqueous Solvents at Pressures Less Than 200 KPA. In IUPAC Solubility Data Series; Pergamon Press: Oxford, UK, 1992; Volume 50. [CrossRef]

55. Matthews, M.J.; Pimenta, M.A.; Dresselhaus, G.; Dresselhaus, M.S.; Endo, M. Origin of dispersive effects of the Raman D band in carbon materials. Phys. Rev. B 1999, 59, R6585-R6588. [CrossRef]

56. Wang, Y.; Alsmeyer, D.C.; McCreery, R.L. Raman spectroscopy of carbon materials: Structural basis of observed spectra. Chem. Mater. 1990, 2, 557-563. [CrossRef]

57. Jensen, G.C.; Krause, C.E.; Sotzing, G.A.; Rusling, J.F. Inkjet-printed gold nanoparticle electrochemical arrays on plastic. Application to immunodetection of a cancer biomarker protein. Phys. Chem. Chem. Phys. 2011, 13, 4888-4894. [CrossRef] [PubMed]

58. Singh, M.; Haverinen, H.M.; Dhagat, P.; Jabbour, G.E. Inkjet Printing-Process and Its Applications. Adv. Mater. 2010, 22, 673-685. [CrossRef] [PubMed]

59. Li, Y.; Rizzo, A.; Mazzeo, M.; Carbone, L.; Manna, L.; Cingolani, R.; Gigli, G. White organic light-emitting devices with CdSe/ZnS quantum dots as a red emitter. J. Appl. Phys. Phys. 2005, 97, 113501. [CrossRef]

60. Talapin, D.V.; Lee, J.; Kovalenko, M.V.; Shevchenko, E.V. Prospects of Colloidal Nanocrystals for Electronic and Optoelectronic Applications. Chem. Rev. 2010, 110, 389-458. [CrossRef] [PubMed] 